\title{
NUMERICAL SIMULATION OF INTERACTIONS BETWEEN WATER WAVES AND A MOORED-FLOATING BREAKWATER
}

\author{
Tobias Martin ${ }^{1}$, Arun Kamath ${ }^{1}$, Hans Bihs ${ }^{1}$
}

\begin{abstract}
Floating breakwaters offer a cost-efficient alternative to common emerged rubble mound breakwaters in deep water coastal areas or harbours with poor seabed conditions. Often, simple box type structures with a suitable mooring system are considered. The motion of the moored box and the resulting tension forces in the cables have to be known during the design process. The accurate determination of these properties is therefore of high significance to configure a safe and economical design. In this paper, a numerical model for determining the influence of the mooring system on floating breakwater dynamics is developed and incorporated into the open-source CFD solver REEF3D.
\end{abstract}

Keywords: Mooring Modelling, Floating Structures, Fluid-Structure Interaction, CFD

\section{INTRODUCTION}

The safety of floating breakwaters has to be guaranteed by an appropriate mooring system. The mooring design has to provide enough strength to withstand the hydrodynamic loads on the structure while restraining its motion. Therefore, the accurate simulation of the moored-floating breakwater are of highly relevancy to construct a safe and economical design.

The calculation of the position of the mooring system often arises from the application of the catenary equation for cables. This analytical approach neglects hydrodynamic effects of the flow on the structure and is just valid in a certain range of shapes. In case of rather taut cables, the catenary form is no longer valid and an alternative approach based on a discrete model has to be considered. To simplify this, a single model which is suitable for slack and taut configurations is preferable. A discretisation of the problem has to be applied due to the underlying non-linear system of equations. There are several models which are based on splitting the cable in finite differences or elements (Huang, 1994; Aamo and Fossen, 2001). In the lumped mass method (Hall and Goupee, 2015), simple truss or spring elements are applied. The knot positions is then found in each time step by solving the force equilibria at each mass point. This approach shows good results for dynamic and static problems but generally lacks the possibility to physically correlate tension forces and deformation of the elements. In order to incorporate this necessary connection between the elements at any time, the tension element method (TEM) was developed (Leitzke, 1983; Hackmann, 1983). The method is limited to quasi-static cases which is appropriate for cases where the exact motion of the cable is not of interest.

The presented mooring model is implemented in the open-source CFD code REEF3D (Bihs et al., 2016). The code has been validated for a wide range of coastal and marine applications like breaking waves kinematics (Kamath et al., 2017b, 2015, 2017), sloshing (Grotle et al., 2017), sediment transport (Ahmad et al., 2018) and floating structures (Bihs and Kamath, 2017).

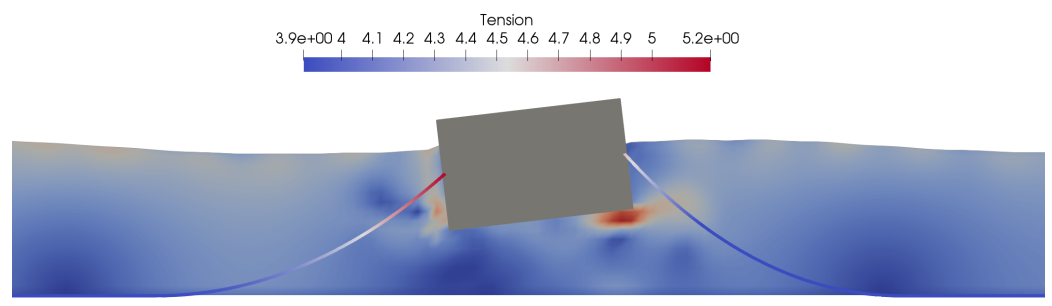

Figure 1: Moored-floating breakwater in a wave trough situation.

\footnotetext{
${ }^{1}$ Department of Civil and Environmental Engineering, NTNU, Trondheim, Norway
} 


\section{NUMERICAL MODEL}

The continuity equation and conservation equations of momentum for incompressible fluids

$$
\begin{aligned}
\frac{\partial u_{i}}{\partial x_{i}} & =0 \\
\frac{\partial u_{i}}{\partial t}+u_{j} \frac{\partial u_{i}}{\partial x_{j}} & =-\frac{1}{\rho} \frac{\partial p}{\partial x_{i}}+\frac{\partial}{\partial x_{j}}\left(v \cdot\left(\frac{\partial u_{i}}{\partial x_{j}}+\frac{\partial u_{j}}{\partial x_{i}}\right)\right)+g_{i}
\end{aligned}
$$

are solved in the computational domain. Here, $u_{i}$ is the velocity components, $\rho$ is the fluid density, $p$ is the pressure, $v$ is the kinematic viscosity and $\vec{g}$ represents the gravity acceleration vector. In the presented calculations, a Reynolds-averaged Navier-Stokes (RANS) approach is incorporated by introducing timeaveraged fluid properties and adding turbulent viscosity to $v$. This additional viscosity is modelled with a $k$ - $\omega$ model including an additional source term for the free surface (see Bihs et al. (2016) for details). The spatial domain is divided into uniform cells of the same length. System (1) is then solved in a staggered manner using finite difference methods (FDM) to avoid decoupling of pressure and velocity. Convection terms are discretised using the fifth-order accurate weighted essentially non-oscillatory (WENO) scheme (Jiang and Shu, 1996) adapted to non-conservative terms (Zhang and Jackson, 2009). Chorin's projection method for incompressible flows (Chorin, 1968) is used for solving the resulting system of algebraic equations. First, a non-divergence free velocity field is calculated employing the third-order accurate Total Variation Diminishing (TVD) Runge-Kutta scheme (Shu and Osher, 1988). Then, the pressure is calculated from the solution of a Poisson equation which is found using a fully parallelized BiCGStab algorithm (van der Vorst, 1992). Finally, the velocities are corrected with the updated pressure such the conservation of mass equation is fulfilled. The free surface is represented implicitly by the zero level set of a smooth signed distance function. It is defined by the closest distance of each point to the interface (Osher and Sethian, 1988). The function is captured in time and space using the linear advection equation. The convection term is discretised by a fifth-order accurate Hamilton-Jacobi WENO method (Jiang and Peng, 2000). The level set function is reinitialized after each time step in order to conserve the signed distance property (Sussman et al., 1994).

\section{Floating Algorithm}

Floating bodies are defined by the location of their centre of gravity and their orientation in the inertial coordinate system described by the three Euler angles $\phi, \theta$ and $\psi$. The translational motions are described by Newton's second law and can be calculated analytically. A coordinate transformation given by Fossen (1994) is applied to simplify the calculation of the rotational motion. Using the principal axes system of the body, the inertia tensor reduces to its main diagonal, and the rotatory motions are given by the Euler equations (Fossen, 1994)

$$
\begin{aligned}
& I_{x} \ddot{\xi}_{1}+\dot{\xi}_{2} \dot{\xi}_{3} \cdot\left(I_{z}-I_{y}\right)=M_{1, \vec{\xi}}, \\
& I_{y} \ddot{\xi}_{2}+\dot{\xi}_{1} \dot{\xi}_{3} \cdot\left(I_{x}-I_{z}\right)=M_{2, \vec{\xi}}, \\
& I_{z} \ddot{\xi}_{3}+\dot{\xi}_{1} \dot{\xi}_{2} \cdot\left(I_{y}-I_{x}\right)=M_{3, \vec{\xi}},
\end{aligned}
$$

which are solved explicitly using the second-order accurate Adams-Bashforth scheme. A weak coupling between fluid and structure is applied. It provides a stable model with accurate results for various applications as could be shown in previous publications (Bihs and Kamath, 2017; Kamath et al., 2017a).

\section{Mooring Algorithm}

The discretisation of the mooring lines is shown in Fig. 2. Each line is divided into a finite number of straight but elastic bars $\vec{f}$. Between the bars, $N$ mass points $P$ are defined. The tension forces $F_{T}$ act at $P$ in the direction of the adjacent bars. All external forces are concentrated on $P$. In this paper, just the gravitational force $\vec{F}_{G}$ is incorporated which is valid for heavy mooring systems. Further, a flexible system is assumed and time dependencies are neglected. Under these assumptions, the solution for the shape of the line and the distribution of tension forces can be found by solving a linear system of equations. This system results from the force equilibria

$$
\vec{f}^{(v+1)} F_{T}^{(v+1)}-\vec{f}^{(v)} F_{T}^{(v)}=-\vec{F}_{G}^{(v)}
$$


at the inner knots $P^{v}$. Here, ${\overrightarrow{F_{G}}}^{(v)}$ is the approximated gravity force from an uniformly distributed mass of the adjacent bars

$$
{\overrightarrow{F_{G}}}^{(v)}=q \vec{g} \cdot\left(\frac{l^{(v)}+l^{(v+1)}}{2}\right)
$$

with $q$ the specific material weight per length in water and $\vec{g}$ the gravity vector pointing in negative $z$-direction.

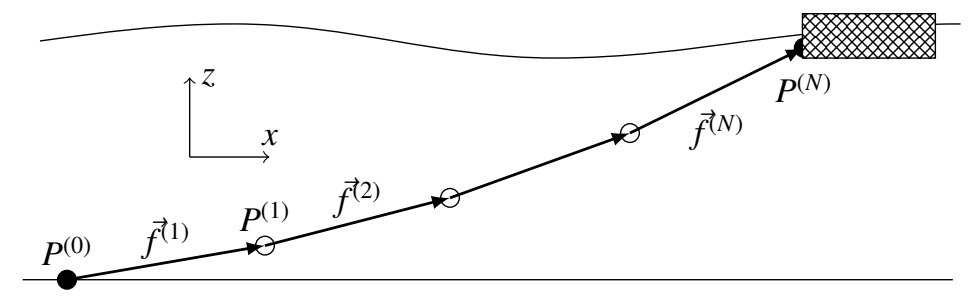

Figure 2: Discrete mooring line: mass points (black points), bars (vectors).

The resulting system is undetermined because the number of bars exceeds the number of inner knots. It is closed by adding a geometrical constraint which is the physical coherence of the mooring line during the deformation. The resulting system of equations can be written in an appropriate way for obtaining the matrix of bar vectors as the solution matrix. Here, the system matrix, containing the unknown tension forces, reduces significantly if comparing to a Newton-Raphson. It results in an improved runtime performance. Since the unknowns are separated, and the system has to be corrected iteratively using the intermediate results until convergence has been reached. After each iteration, the lengths of the bar vectors have to equal one by definition. Therefore, a correction step has to be performed for the system matrix before the loop proceeds. The algorithm stops in case of reaching a predefined criterion for the residuals which corresponds to the conservation of all bar unit vectors within a certain tolerance. The calculated forces of the mooring lines on the body are included in the floating algorithm in an explicit manner.

\section{RESULTS}

\section{Validation of the Mooring Model}

The principal working of the mooring model is shown using an elastic catenary solution (Faltinsen, 1990). This analytical solution represents the physical shape of a chain between two fixed points. Fig. 3 shows the convergence of the TEM to this analytical solution with increasing number of elements $N$. The $L_{2}$-norm of the error in $z$-direction decreases from 0.009 for $N=3$ to 0.004 for $N=50$.

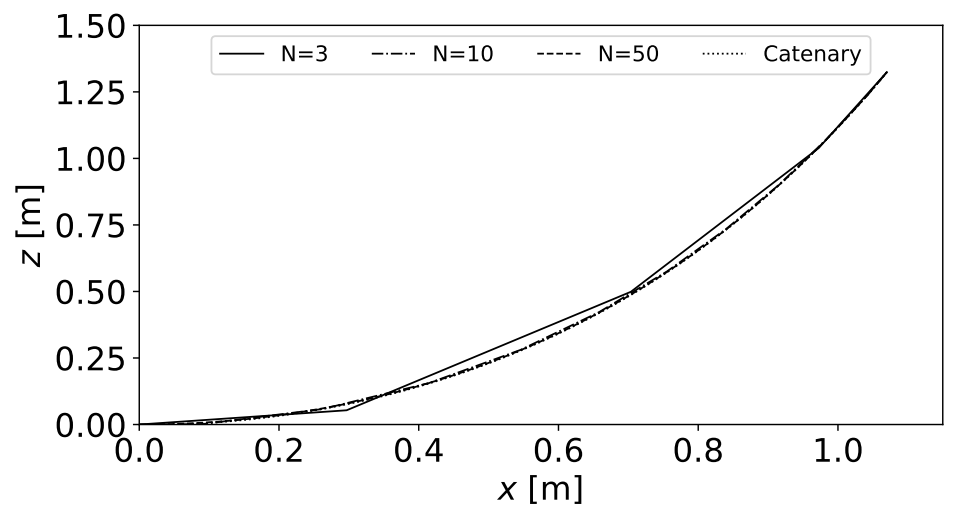

Figure 3: Numerical Solution of the mooring model using $N$ elements in comparison to the elastic catenary solution. 


\section{Validation of the Floating Algorithm}

First, the developed floating algorithm is validated for single heave and pitch decay motions. A numerical heave decay test for a cylinder of diameter $0.1524 \mathrm{~m}$ and density $500 \mathrm{~kg} / \mathrm{m}^{3}$ is compared to experimental data (Yang, 2018). The cylinder is partially submerged with its centre located at $0.0254 \mathrm{~m}$ above the water surface. As soon as the body is released, a heave motion with decreasing amplitude can be observed over time. In $4 \mathrm{a}$ the results for three different cell sizes are compared to the experiments. The coarsest grid overpredicts the first peak while the finer grids reproduce the experimental data in amplitude and frequency properly.

A numerical pitch decay test for a box of length $0.3 \mathrm{~m}$, height $0.1 \mathrm{~m}$ and density $856 \mathrm{~kg} / \mathrm{m}^{3}$ compared to experimental data (Yang, 2018) is investigated. The box is partially submerged with its centre located at the water surface but rotated by 15०. The pitch motion with decreasing amplitude is observed over time. In $4 \mathrm{~b}$ the results for three different cell sizes are compared to the experiments. All grid resolutions result in slightly over-predicted amplitudes but a very good agreement with the experiments with regards to frequencies of the motion.

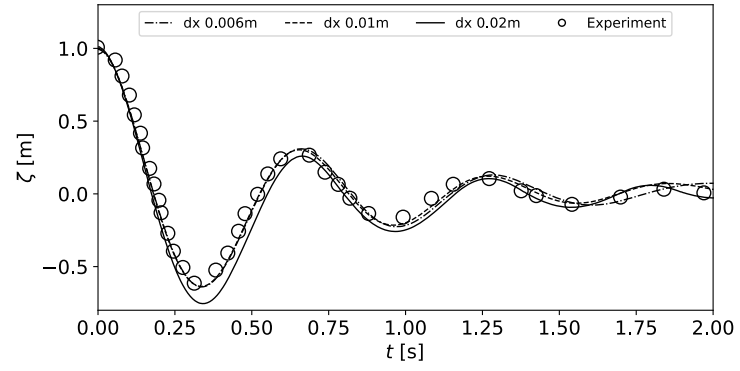

(a) Heave decay test: Heave amplitude over time.

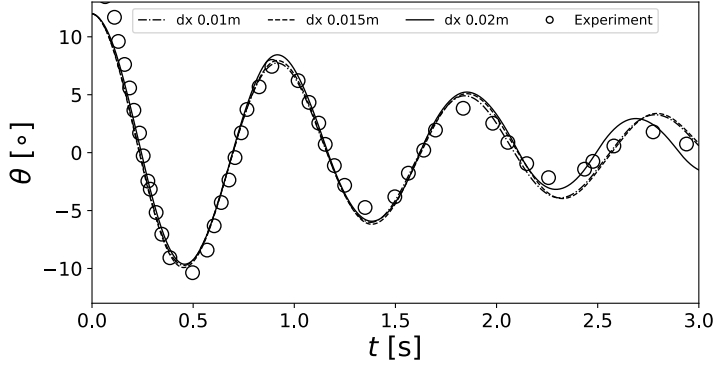

(b) Pitch decay test: Pitch amplitude over time.

Figure 4: Numerical and experimental results for the heave (a) and pitch (b) decay tests.

\section{Moored-Floating Breakwater in Waves}

The effect of mooring on the motion of a floating breakwater is presented next. The 2D numerical setup is taken from experiments of a free-floating breakwater (Ren et al., 2015). The tank's dimensions are $20 m \times 0.8 m$ and the water depth is $0.4 m$ (Fig. 5). A rectangular breakwater of $0.30 m \times 0.2 m$ and density $500 \mathrm{~kg} / \mathrm{m}^{3}$ is placed in the tank at $(x, z)=(7.0 \mathrm{~m}, 0.4 \mathrm{~m})$. Second-order Stokes waves of $h=0.02 \mathrm{~m}, T=1.2 \mathrm{~s}$ and $\lambda=1.336 \mathrm{~m}$ are generated at the inlet using a relaxation method. Wave reflections are prevented at the outlet by using a numerical beach zone. The chosen mooring system consists of two lines fixed to the body at the free surface. Both lines have a length of $1.9 \mathrm{~m}$ and a thickness of $0.004 \mathrm{~m}$. Two configurations with different specific material weight in water of $0.25 \mathrm{~kg} / \mathrm{m}$ and $1.5 \mathrm{~kg} / \mathrm{m}$ are considered. The results in Fig. 6 show the heave, surge and pitch motions of the free-floating and moored-floating breakwater between $t=8 s$ and $t=14 s$.

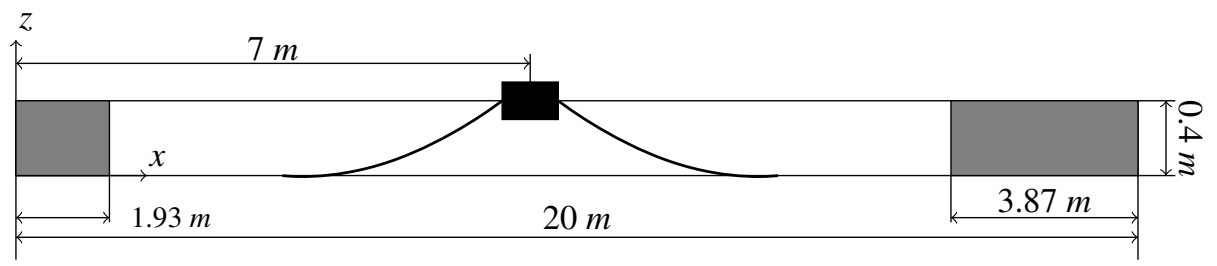

Figure 5: Setup for the 2D moored-floating breakwater in a numerical wave tank.

As can be seen in Fig. 6a, the heave motion is just slightly influenced by the mooring system in both amplitude and frequency. The rather flat angles between line and body at the attachment points result in small vertical reaction forces which influence this motion. It is however noticeable that the draft of the breakwater increases due to the additional weight of the system, which is obviously even more noticeable 
for the heavier configuration. Fig. 6b shows the effect of mooring on the surge motion of the breakwater. The free-floating breakwater moves in x-direction over time due to Stokes drift. In contrast, the mooring systems prevent this motion because of large horizontal reaction forces. Additionally, the amplitude of the motion decreases with the increasing weight. Similarly, the pitch motion is reduced by the counteracting tension forces of the mooring systems (see Fig.6c).

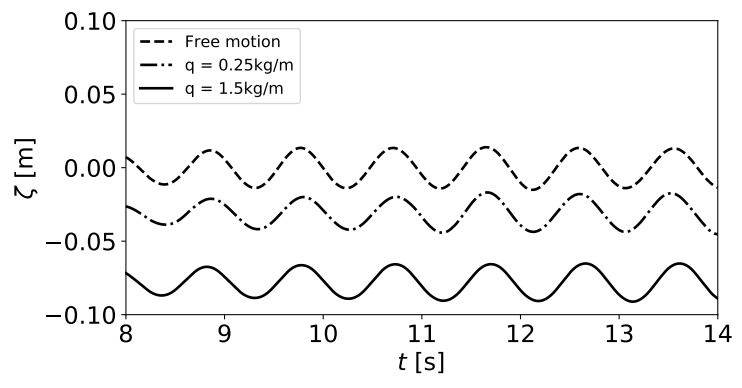

(a) Heave motion.

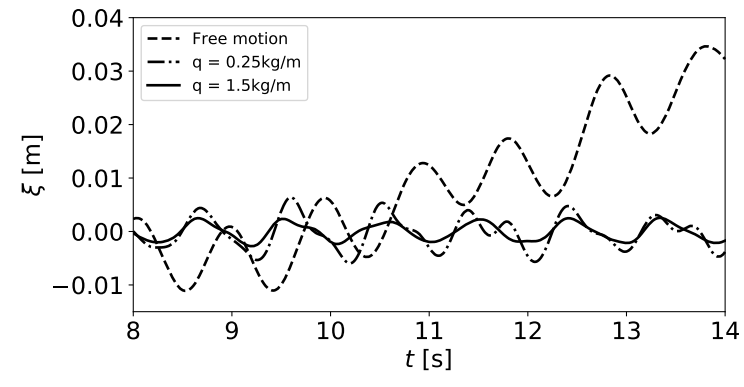

(b) Surge motion.

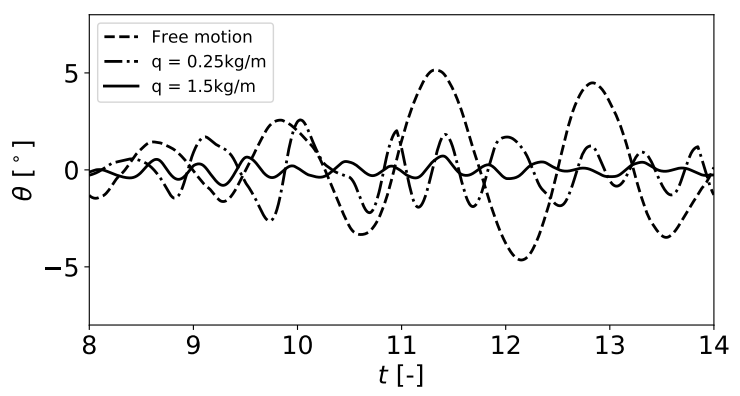

(c) Pitch motion.

Figure 6: Numerical results of the moored-floating breakwater in comparison to the free-floating breakwater.

\section{Analysis of the Motion of a Moored-Floating Breakwater}

The sensitivity of the breakwater's motion with respect to different mooring systems shall be emphasised. For this purpose, different mooring systems are connected to the floating breakwater and waves of the same height but different lengths are imposed in the numerical tank. The dimensions of the tank and breakwater are taken from above. Further, the shown mooring configurations with different material weights are considered but with a slightly increased length resulting in a different angle between line and body at the mooring point. As results, the maximum amplitudes and frequencies of the heave and pitch motion for wave lengths between $\lambda=0.2 \mathrm{~m}$ and $\lambda=3.0 \mathrm{~m}$ are compared to the free-floating breakwater (see Fig. 7). All computations are executed with cell size $\Delta x=0.01 \mathrm{~m}$.

For the heave motion in Fig. 7a, the decrease in amplitude increases over the wavelength up to $\lambda=1.5 \mathrm{~m}$ and stays constant afterwards. While for small wavelengths the amplitude is similar, the frequency of the motion changes due to the mooring forces (Fig. 7b). The results for the pitch motion are similarly. The frequency changes just for small wavelengths while the amplitudes change for larger wavelengths. It might be also noticed that for certain wavelengths, the maximum pitch amplitude increases for a light mooring system but decreases for a heavy system. This results in an overall different response function of the moored-floating body. At the same time, the global maximum of the pitch motion is smaller if mooring is applied due to the additional damping.

\section{CONCLUSION}

The open-source CFD solver REEF3D is applied to simulate the interaction of waves with a mooredfloating breakwater. The presented numerical mooring model provides an accurate solution for quasi-static applications. A toolbox for investigating complicated fluid-structure interactions including mooring could 


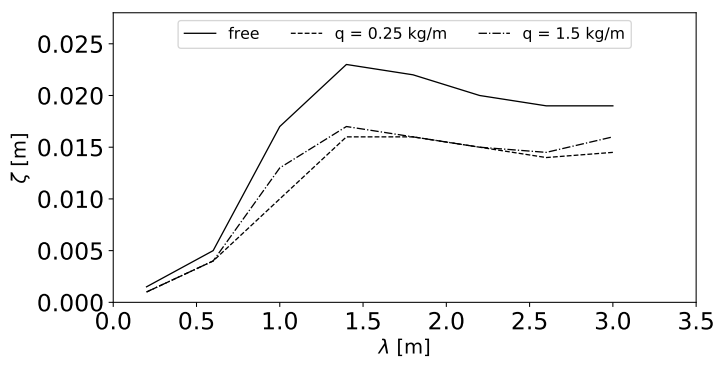

(a) Maximum heave amplitudes over $\lambda$.

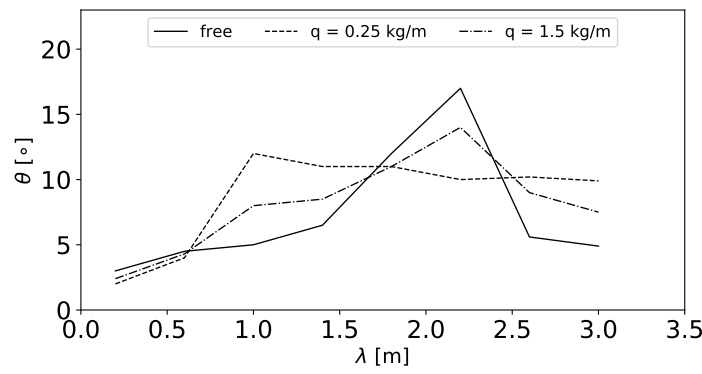

(c) Maximum pitch amplitudes over $\lambda$.

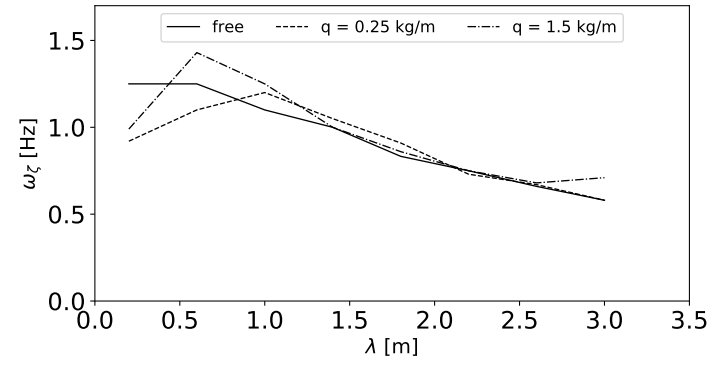

(b) Maximum heave frequencies over $\lambda$.

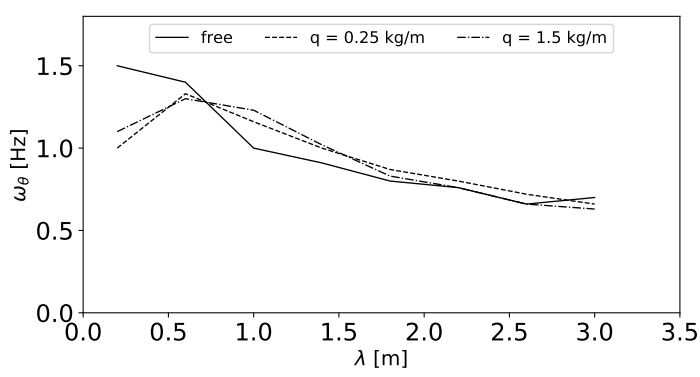

(d) Maximum pitch frequencies over $\lambda$.

Figure 7: Numerical results for the maximum amplitudes and frequencies in heave and pitch for different wave lengths.

be presented by coupling the developments to the accurate wave modelling of REEF3D. The results verify the implementation of the discrete mooring model and indicate its validity for simulating dynamic mooring systems. The influence of mooring on a floating breakwater was investigated. It keeps the body in position and reduces rolling. The analysis of the motion for different wavelengths emphasises the importance of a careful selection of the cable properties. These properties play a major role for the response of the breakwater and the occurring tension forces in the mooring system. If the typical wave conditions are known, the presented mooring model can be used for getting useful hints for an optimal design of the mooring system.

\section{ACKNOWLEDGEMENTS}

The authors are grateful for the grants provided by the Research Council of Norway under the HAVBRUK2 project (No. 267981). This research was supported in part with computational resources at NTNU provided by The Norwegian Metacenter for Computational Sciences (NOTUR, http://www.notur.no) under project no. NN2620K.

\section{References}

O. Aamo and T. Fossen. Finite Element Modelling of Moored Vessels. Mathematical and Computer Modelling of Dynamical Systems, Volume 7(1):47-75, 2001.

N. Ahmad, H. Bihs, D. Myrhaug, A. Kamath, and Ø. A. Arntsen. Three-dimensional numerical modelling of wave-induced scour around piles in a side-by-side arrangement. Coastal Engineering, Volume 138: $132-151,2018$.

H. Bihs and A. Kamath. A combined level set/ghost cell immersed boundary representation for floating body simulations. Int. J. Numer. Meth. Fluids, Volume 83:905-916, 2017.

H. Bihs, A. Kamath, M. A. Chella, A. Aggarwal, and Ø. Arntsen. A new level set numerical wave tank with improved density interpolation for complex wave hydrodynamics. Computers $\mathcal{E}$ Fluids, Volume 140: 191-208, 2016. 
A. Chorin. Numerical solution of the Navier-Stokes equations. Mathematics of Computation, Volume 22: 745-762, 1968.

O. Faltinsen. Sea Loads on Ships and Offshore Structures. Cambridge University Press, Cambridge, 1990.

T. Fossen. Guidance and Control of Ocean Vehicles. John Wiley \& Sons: Chichester, England, 1994.

E. Grotle, H. Bihs, and V. Æsøy. Experimental and numerical investigation of sloshing under roll excitation at shallow liquid depths. Ocean Engineering, Volume 138:73-85, 2017.

W. Hackmann. Mathematische Begründung von Verfahren zur Berechnung von Form und Zugkraft in Fadenzugsystemen. ZAMM, Volume 63:173-184, 1983.

M. Hall and A. Goupee. Validation of a lumped-mass mooring line model with DeepCwind semisubmersible model test data. Ocean Engineering, Volume 104:590-603, 2015.

S. Huang. Dynamic analysis of three-dimensional marine cables. Ocean Engineering, Volume 21:587-605, 1994.

G. Jiang and D. Peng. Weighted ENO schemes for Hamilton Jacobi equations. SIAM Journal of Scientific Computing, Volume 21:2126-2143, 2000.

G. Jiang and C. Shu. Efficient implementation of weighted ENO schemes. Journal of Computational Physics, Volume 126(1):202-228, 1996.

A. Kamath, M. A. Chella, H. Bihs, and $\varnothing$. Arntsen. Energy transfer due to shoaling and decomposition of breaking and non-breaking waves over a submerged bar. Engineering Applications of Computational Fluid Mechanics, Volume 11 (1):450-466, 2017.

A. Kamath, M. A. Chella, H. Bihs, and Ø. A. Arntsen. Evaluating wave forces on groups of three and nine cylinders using a 3D numerical wave tank. Engineering Applications of Computational Fluid Mechanics, Volume 9:343-354, 2015.

A. Kamath, H. Bihs, and Ø. A. Arntsen. Study of Water Impact and Entry of a Free Falling Wedge Using Computational Fluid Dynamics Simulations. J. Offshore Mech. Arct. Eng., Volume 139(3), 2017 a.

A. Kamath, M. A. Chella, H. Bihs, and Ø. A. Arntsen. Energy transfer due to shoaling and decomposition of breaking and non-breaking waves over a submerged bar. Engineering Applications of Computational Fluid Mechanics, Volume 11(1):450-466, $2017 \mathrm{~b}$.

H. Leitzke. Berechnung von Form und Kräften biegeschlaffer, räumlicher Zugsysteme. Ph.D. thesis, University of Rostock, 1983.

S. Osher and J. Sethian. Fronts propagating with curvature-dependent speed: Algorithms based on Hamilton-Jacobi formulations. Journal of Computational Physics, Volume 79:12-49, 1988.

B. Ren, M. He, P. Dong, and H. Wen. Nonlinear simulations of wave-induced motions of a freely floating body using WCSPH method. Applied Ocean Research, Volume 50:1-12, 2015.

C. Shu and S. Osher. Efficient implementation of essentially non-oscillatory shock-capturing schemes. Journal of Computational Physics, Volume 77(2):439-471, 1988.

M. Sussman, P. Smereka, and S. Osher. A level set approach for computing solutions to incompressible two-phase flow. Journal of Computational Physics, Volume 114:146-159, 1994.

H. van der Vorst. BiCGStab: A fast and smoothly converging variant of Bi-CG for the solution of nonsymmetric linear systems. SIAM Journal of Scientific Computing, Volume 13:631-644, 1992.

L. Yang. One-fluid formulation for fluid-structure interaction with free surface. Computer Methods in Applied Mechanics and Engineering, Volume 332:102-135, 2018.

J. Zhang and T. L. Jackson. A high-order incompressible flow solver with WENO. Journal of Computational Physics, Volume 228:146-159, 2009. 